\title{
Climate Crisis and the Democratic Prospect. Participatory Governance in Sustainable Communities
}

Frank Fischer

Oxford 2017: Oxford University Press, 352 Seiten.

Iris Kunze

Universität für Bodenkultur Wien, Austria

E-Mail: iris.kunze@boku.ac.at
Frank Fischer fordert mit dem vorliegenden Buch nicht nur dringend mit demokratischen, partizipativen Methoden politisch auf die ökologische, insbesondere die Klimakrise aktiv zu reagieren, er führt auch zahlreiche Beispiele ein, in denen dies bereits in Ansätzen und in lokalen Kontexten passiert. Damit verbindet sein Buch die bisher kaum behandelte Verbindung zwischen globaler Umweltpolitik, Demokratietheorie und Graswurzelbewegungen, um daraus Fragen und mögliche Lösungsansätze für die Klimakrise - die auch eine politische Krise ist - zu erörtern. Im Kontext der Politikwissenschaften wagt er pionierhaft einen gut begründeten Appell an die etablierten politischen Systeme, Vorreiterinitiativen aus der Zivilgesellschaft in ihrem Potential als professionelle Transformationsakteure ernst zu nehmen und in den politischen Steuerungsprozess einzubeziehen.

In den Eingangskapiteln bemängelt Fischer die träge Handlungsfähigkeit demokratisch-bürokratischer Politik angesichts des drängenden Problems des Klimawandels, weshalb er seine Hoffnung in zivilgesellschaftliche Initiativen setzt. Damit deren Erfahrung und Expertise in die Politik einfließen kann, macht er ein Konzept von Bürgerbeteiligung („participatory governance“) stark, das sich an lokalen Spezifika orientiert und sich auf politische Entscheidungsprozesse einlässt. Immer geht es Fischer um die konkrete Umsetzung, die mittels zahlreicher Beispiele erläutert wird.

Aus seinen weltweit empirischen Fallbeispielen folgert er die Wichtigkeit, dass Methoden der Organisation und Moderation für partizipatorische Prozesse verstärkt gelernt und kultiviert werden müssen. Er stellt zwei Beispiele von umweltpolitischer Teilhabe näher vor: erstens die gemeinschaftliche Waldbewegung in Nepal. Dieser Fall gelang, weil involvierte Forscher den Prozess feder- führend angestoßen und begleitet haben und vermutlich die Zivilgesellschaft nicht die Kompetenzen oder Akzeptanz gegenüber der Politik hätte aufbringen können. Zweitens führt er die weltweite Ökodörfer-Bewegung als ein seltenes Beispiel partizipativer Umweltpolitik ein, weil Ökodörfer diese Werte in der Lebenspraxis umsetzen. Was Fischer nicht ausführt, ist, dass man bei diesen Projekten nicht mehr von politischer Partizipation sprechen kann, sondern vielmehr von Selbstorganisation. Vorgestellte Beispiele wie das Ökodorf Sieben Linden sind durch seine Bewohner selbst durch langwierige Konsensentscheidungsprozesse geplante Gemeinschaften. Gemeinschafts- und Vertrauensbildung sind Grundlage für die Einigung auf gemeinsame Werte sowie finanzielles und soziales Engagement für die Gemeinschaft, was schließlich zu einer ökologischen Lebenspraxis führt, die durch rechtliche Rahmenbedingungen wie gemeinnützigen, gemeinschaftlichen Besitz in Form von Genossenschaft oder Verein mit ökologisch ausgerichteten Werten organisiert und konsolidiert wird. Diese Projekte erfordern daher zahlreiche Fähigkeiten von ihren Mitgliedern, wie die Folgen des eigenen Handelns nicht nur persönlich, sondern auch im Kontext einer Gemeinschaft überblicken und abschätzen zu können, dabei vor allem die Fähigkeit Verantwortung zu übernehmen. Hier stellt sich einerseits die Frage, ob die Sozialisation und Bildung die Bürger ausreichend ausstattet. Andererseits fehlen den politischen Akteuren die Methoden und das Vertrauen, die Bürger in die Entscheidungsprozesse und Verantwortungsübernahme einzubeziehen.

Fischer führt weitere Beispiele ein, in denen zivilgesellschaftliche Akteure sich zu Graswurzelbewegungen zusammenschließen, um Freiräume der Selbstorganisa- 
tion für umweltverantwortliches Handeln zu schaffen, wie "transition towns" und "repair cafes". Gerade die Globalisierung hat ein Revival der lokalen Ebene hervorgerufen, weil erst hier wieder Demokratie umgesetzt werden kann. Fischer untermauert seine Thesen nicht nur mit aktuellen, sondern auch historischen Fallbeispielen und Theoretikern von Dezentralisierung oder Bioregionalismus. Dass er dabei Rudolf Bahros Theorien ausführt, lässt den Fakt vermissen, dass dieser eine Öko-Kommune angestoßen hat, die in der Praxis letztlich kläglich an mangelnder Integration und unbewussten Machtstrukturen gescheitert ist (Kunze 2009), im Gegensatz zu vielen anderen Öko-Gemeinschaften ohne einen charismatischen Gründer oder eine radikale Theorie, aber stattdessen mit einem organischen Gemeinschaftsbildungsprozess wie Sieben Linden oder Schloss Tempelhof (ibid, Kunze/Avelino 20I5). Dieses Fakt untermauert die leider nur nebenbei erwähnte These, dass der Wandel der sozialen und kulturellen Dimension eine wesentliche Grundlage für die Realisierung einer partizipativen Umweltdemokratie („participatory environmental governance") ist.

Im letzten Kapitel macht Fischer sich noch einmal auf die Suche nach Ansätzen in der politischen Theorie, die seine empirischen Beobachtungen und Schlussfolgerungen aufgreifen könnten. Fischer verweist auf das Konzept der assoziativen Demokratie, denn er befürchtet Konfliktpotential, wenn staatliche Politik nicht die kritischen Bewegungen aus der Zivilgesellschaft anerkennen. Assoziative Demokratie verwendet ein Selbstverwaltungsprinzip, das nicht nur im institutionalisierten politischen Rahmen, sondern auch in der Wirtschaft und Zivilgesellschaft angewendet werden kann. Es basiert auf Solidarität und gemeinsamen Einigungen bei der Akzeptanz von Verschiedenheit und Diversität. Fischer schließt mit einer zusammenfassenden Diskussion. Die Aussage, dass die beste Lösung zur Verhinderung des Klimawandels eine volle sozial-ökologische Transformation in Richtung nachhaltiger Lebensweise wäre, sticht befreiend als Maximal-Vision hervor. Wenn das nicht angegangen wird, droht nach Fischer militärisches Eingreifen, um die durch Naturkatastrophen dramatischen Umstände wie Ressourcen- und Nahrungsmittelknappheit zu bewältigen. Auch wenn an einigen Stellen die Dringlichkeit der Klimakrise betont wird, bleibt der Ton des Buches grundsätzlich hoffnungsvoll, und es wird betont, dass es bereits viele Initiativen und Projekte im Kleinen gibt, die nur darauf warten von der größeren Politik ernst genommen und aufgegriffen zu werden.

\section{Literatur}

Kunze, Iris (2009), Soziale Innovationen für zukunftsfähige Lebensweisen. Gemeinschaften und Ökodörfer als experimentierende Lernfelder für sozialökologische Nachhaltigkeit. Dissertation. Münster: Ecotransfer-Verlag,

Kunze, Iris/Avelino, Flor (2015), Social innovation and the Global Ecovillage Network (TRANSIT research report), TRANSIT: EU SSH.2OI3.32-I Grant agreement no: 613169 